FOLIA

Amazónica

Revista del Instituto de Investigaciones

de la Amazonía Peruana

\title{
COMPOSICIÓN QUÍMICA DE LAS HOJAS Y SEMILLAS DE SACHA INCHI (Plukenetia volubilis L.); OLEAMIDA
}

\author{
Diego CORTES ${ }^{1}$, Nuria CABEDO ${ }^{1}$, Laura VILA ${ }^{1}$, Claudia SOLANO², \\ Juan BENAVIDES ${ }^{3}$, Diana FLORES ${ }^{4}$
}

1 Universidad de Valencia, Facultad de Farmacia. Vicent Andrés Estellés s/n, Burjassot, 46100, Valencia, España.

2 Comisión de Promoción del Perú para la Exportación y el Turismo - PROMPERU. Calle Uno Oeste №50, Urb. Corpac, San Isidro. Lima, Perú.

3 Asociación Promotora Del Desarrollo De Sacha Inchi- PRO-SACHA INCHI. Calle Uno de la Av El Sol, Manzana O, Lote 5a, Villa El Salvador. Lima, Perú.

4 Consultor. Jr. Monte Real 433-303, Chacarilla del Estanque, Surco. Lima, Perú.

* Correo electrónico: diego.m.cortes@uv.es

\section{RESUMEN}

El estudio se realiza con el objeto de identificar el tipo de alcaloide, protoalcaloide o pseudoalcaloide, que puedan ser cuantificables en las hojas y en la torta de semillas tostadas y desengrasadas de la especie Plukenetia volubilis L. y demostrar que su presencia no representa un riesgo para la salud. La metodología utilizada está basada en protometría de acuerdo a la Farmacopea Francesa y Europea, así como la utilización de técnicas de UHPLC-MS/MS y GC-MS que han permitido identificar y cuantificar un compuesto endógeno que revela las características de la cis-9-octadecenamida (oleamida), estructura similar a la anandamida; ligando endógeno de receptores cannabinoides en concentraciones de 0,0015\% en hojas y $0,0004 \%$ en semillas. No habiendose detectado alcaloides que puedan afectar la seguridad alimentaria.

PALABRAS CLAVE: ácido graso endógeno, cis-9-octadecenamida. 


\title{
CHEMICAL COMPOSITION OF SACHA INCHI LEAVES AND SEEDS (Plukenetia volubilis L.); OLEAMIDE
}

\begin{abstract}
This study is carried out to identify the type of alkaloid, protoalkaloid or pseudoalkaloid, that can be quantified in leaves and in toasted and defatted seed cake of Plukenetia volubilis L. and that their presence does not represent a health risk. The methodology used is based on protometry according to the French and European Pharmacopoeia, as well as the use of UHPLC-MS / MS and GC-MS techniques that have made it possible to identify and quantify an endogenous compound that reveals the characteristics of cis-9 -octadecenamide (oleamide), structure similar to anandamide; endogenous ligand of cannabinoid receptors in concentrations of $(0,0015 \%)$ in leaves and $(0,0004 \%)$ in seeds. No alkaloids have been detected that could affect food safety.

KEYWORDS: cis-9-octadecenamide, endogenous fatty acid.
\end{abstract}




\section{INTRODUCCIÓN}

La semilla de sacha inchi (Plukenetia volubilis L.), ha sido encontrada en las tumbas incaicas de la costa peruana de la cultura Mochica, Chimú, probablemente cultivada desde hace 3000 - 5000 años; mencionada en la obra "Los Comentarios Reales" por Inca Garcilaso de la Vega (1609) como "inchic". Descrita por Linnaeus en 1753 y reportada por Mcbride (1951) en la obra "Flora of Perú". Plukenetia volubilis pertenece a la familia Euphorbiaceae (Gillespie, 1993), tradicionalmente conocida como: maní del inca, sacha yuchi, sacha yuchiqui (Antúnez de Mayolo, 1981; Brack, 1999; Doherty, 2007), considerada planta promisoria por el Convenio Andrés Bello conforme Correa \& Yesid (1992). Posteriormente Dostert et al. (2013) presentaron el estado del conocimiento botánico de siete especies de plantas utilizadas en el Perú, entre ellas Plukenetia volubilis L. o "sacha inchi".

Las semillas son valoradas por su alto contenido de ácidos grasos insaturados, con rendimientos del $41,5 \%$ de acuerdo a Chirinos et al. (2013) y al 50,4\% reportados por: Hamaker et al. (1992) y Follegatti-Romero et al. (2009), incluye $\omega 3, \omega 6$ y $\omega$ 9. El perfil de grasas de esta oleaginosa fue considerada equivalente al aceite de linaza (Linum usitatissimum L.) por la EFSA (European Food Safety Authority) y tiene el estatus GRAS (Generally Recognized as Safe)(2014) por la FDA. Su estabilidad está basada en la presencia de tocoferoles especialmente el $\delta$ y $\gamma$ tocoferol. Fanali et al. (2011), reportaron 126 mg de $\gamma$-tocoferol, $87 \mathrm{mg}$ de $\delta$-tocoferol y $0,4 \mathrm{mg}$ de $\alpha$-tocoferol por $100 \mathrm{~g}$ de aceite; los tocoferoles son considerados potentes compuestos antioxidantes. Además, Fanali et al. (2011) informaron que el contenido fenólico total del aceite de sacha inchi es de $6,20 \mathrm{mg} / 100 \mathrm{~g}$ de aceite, pertenecientes a las clases de polifenoles de alcohol fenílico, flavonoide, secoiridoide y lignina. El aceite de sacha inchi contiene 247 $\mathrm{mg}$ de esteroles por $100 \mathrm{~g}$ de aceite, siendo el $\beta$-sitosterol la mayor parte de los esteroles (56,5\%), seguido del estigmasterol $(27,9 \%)$, campesterol $(7,1 \%)$ y $\Delta 5$-avenasterol $(5,3 \%)$; y menos del $1 \%$ de otros esteroles, incluidos niveles bajos de colesterol $(0,3 \%)$ según los resultados obtenidos por Bondioli et al. (2006).

En relación con el contenido de proteínas en la semilla varia de $25-35 \%$ (Hamaker et al., 1992; Gutierrez et al. 2011), y en semillas desengrasadas de 27-59,1\%, comprende todos los aminoácidos esenciales en cantidades adecuadas en comparación con el patrón recomendado por la FAO/OMS y un contenido ligeramente bajo en lisina y leucina, citado por: Hamaker et al. (1992); Sathe et al. (2002); Follegatti-Romero et al. (2009); Cai et al. (2012); Gutierrez et al. (2011) y Ruiz et al. (2013). Entre los aminoacidos destacan el contenido del triptófano: $44 \mathrm{mg} / \mathrm{g}$ de proteína (Sathe et al., 2002). Además, de un $8,7 \%$ de fibra dietaría de acuerdo con las hojas técnicas de las empresas; los principales minerales presentes que contiene son: potasio (5563,5 ppm), magnesio (3210 ppm) y calcio (2406 ppm) según Gutierrez et al. (2011).

Por otra parte, la familia Euphorbiaceae incluye especies con compuestos considerados tóxicos de acuerdo a Seigler (1994). Asimismo, Antúnez de Mayolo (1981) menciona que las comunidades nativas consumen las semillas de sacha Inchi, tostadas o cocidas debido a su astringencia y sus efectos a nivel digestivo, esta forma de uso también es mencionada por Correa \& Yesid (1992). Las agencias alimentarias a nivel mundial otorgan particular importancia a los niveles de antinutrientes presentes en los alimentos de consumo que puedan ser dañinos para la salud. Entre los antinutrientes reportados en la especie Plukenetia volubilis L. tenemos: saponinas y 
taninos (Ruiz et al., 2013; Mondragón, 2009), con valores inferiores respecto a los reportados para soya por Ruiz et al. (2013). Asimismo, Castillo (2010) menciona la presencia de alcaloides en las hojas y tallos por análisis cualitativo; Castaño et al. (2012) reportan en el tamizaje fitoquímico de extractos de semillas y de hojas la presencia de saponinas, taninos y alcaloides. Srichamnong et al. (2018), establecen la presencia de alcaloides semillas y en hojas frescas y tostadas de P. volubilis L, cultivadas en Tailandia, utilizando el método Shamsa et al., (2008), basado en la reacción con Verde de Bromocresol (BCG) [reacciona con los alcaloides que tienen nitrógeno en su estructura; los alcaloides de amida y amina no reaccionan con este método], encontrando una mayor cantidad de alcaloides en las semillas sin procesar (485 $\pm 35 \mathrm{mg} / \mathrm{kg} \mathrm{DW}$ ) que en las hojas (146 $\pm 7 \mathrm{mg} /$ kg DW). El tratamiento térmico es muy utilizado en la reducción de antinutrientes a excepción de las saponinas que además usa otros métodos, el efecto térmico fue estudiado en sacha inchi por Bueno-Borges (2018). Debido a la capacidad que tiene la lectina o aglutinina de presentar actividad hemolítica este autor realizó ensayos de hemaglutinación para lectinas (Nowak et al., 1976, modificado Mendonça-Franqueiro et al., 2011), no presentando actividad aglutinante. No obstante, los ensayos realizados por Srichamnong et al.(2018), detectaron una cantidad muy baja de lectinas en semillas tostadas, del orden del 0,15 a 0,22 ng/kg DW; las lectinas son afectadas por el proceso térmico. Adicionalmente, Alcivar et al. (2020) reportan la presencia de alcaloides cualitativamente en la torta de la semilla. Sin embargo, en ninguna de las investigaciones, se llega a identificar el tipo de alcaloide presente que podría impedir la comercialización de las semillas tostadas de sacha inchi o si estos no son beneficiosos para la salud.

Los alcaloides son metabolitos secundarios presentes en diferentes especies, tienen diversas clasificaciones, por su origen biogenético tenemos: (a) alcaloides verdaderos (b) protoalcaloide (c) pseudoalcaloide (Hegnauer, 1963).

En ese contexto Matsuura (2015), menciona la presencia de los alcaloides tropánicos en la familia Euphorbiaceae, los cuales están regulados por la European Commission (EC), priorizándose a las familias Brassicaceae, Solanaceae y Erythroxylaceae como fuente de alcaloides tropánicos (EFSA-CONTAM, 2013); sin embargo, la comisión evaluadora europea no descarta su presencia en otras familias. Por otra parte, Dreger (2009) menciona la presencia de alcaloides del tipo pirrolizidinico en la familia Euphorbiaceae. El Panel de contaminantes de la Cadena Alimentaria (EFSA-CONTAM, 2011) encuentra que los alcaloides pirrizolidinicos (AP) por su naturaleza podrían estar presentes en las familias Boraginaceae, Asteraceae, Orchidaceae y Leguminosae [notificación "G/SPS/N/EU/406" de fecha 6 de agosto de 2020]. El contenido de los AP depende de una gran cantidad de factores (especies, órganos de la planta, cosecha, almacenamiento, procedimientos de extracción); en adelante nos enfocamos a la búsqueda de alcaloides, protoalacaloides o pseudoalacaloides en las semillas tostadas y hojas con la finalidad de demostrar la inocuidad del alimento.

\section{MATERIAL Y MÉTODOS}

El Proyecto Innovación y Competitividad para el Agro Peruano-INCAGRO, financió parcialmente el Sub-Proyecto: Obtención de líneas mejoradas de sacha inchi (Plukenetia volubilis L.), a partir del material genético con altos rendimientos y contenidos de omega 3 y omega 6 (CorazónGuivin et al. 2009).

Una de las 12 accesiones de San Martín de alto rendimiento es Plukenetia volubilis L., 
Código Nacional PER000395 procedencia de Pinto Recodo, Provincia de Lamas ubicada en el Departamento de San Martin, cuyo ecotipo comercial es utilizado por las Asociaciones de productores organizados en la cadena de valor desde el 2007, apoyados por el Comité Técnico de Normalización de sacha inchi y sus derivados.

Los "snacks" (bocaditos o semillas tostadas) de sacha inchi (NTP 151.403: 2018), proceden de la Asociación Promotora del Desarrollo de Sacha Inchi - Pro Sacha Inchi, a partir de los cuales, se preparó $300 \mathrm{~g}$ de "torta" (semilla tostada desgrasada); Lote: TORTTSIO-001-20.08.2020. Asimismo, se desecaron y pulverizaron $200 \mathrm{~g}$ de hojas pertenecientes a los mismos predios de la zonas productoras de las semillas.

La metodología para aislar, purificar y cuantificar el contenido de alcaloides se basa en un método gravimétrico y protometria llevados a cabo según la técnica de la Farmacopea Francesa y Europea. Además, se utilizó un equipo UHPLCPDA-MS/MS y GC-MS [Espectrómetro de masas Agilent 5977A con analizador cuadrupolar de baja resolución, con cromatógrafo de gases (Agilent 7890B)], realizados en la Universidad de Valencia, que cuenta con la certificación ISO 9001 como acreditación de calidad.

- Alcaloides en las hojas de sacha inchi: las hojas desecadas y pulverizadas se desengrasaron y se extrajo mediante Soxhlet a polaridad creciente.

- Detección cualitativa de alcaloides: una alícuota de cada uno de los residuos de los extractos Hexánico (A), Diclorometánico (B) y Metanólico (C) son extraídos con $\mathrm{H}_{2} \mathrm{SO}_{4}$ $0,5 \mathrm{~N}$; estas soluciones ácidas son tratadas con metales pesados formándose complejos con los alcaloides por reacción de precipitación. El residuo del extracto $\mathrm{CH}_{2} \mathrm{Cl}_{2}$ (B) previa alcalinización da positivo para alcaloides, asimismo se aplica la técnica de Cromatografia en Capa Fina (CCF).
- Valoración cuantitativa de alcaloides en las hojas: una alícuota del residuo (B), 0,59 g es disuelto en $\mathrm{CH}_{2} \mathrm{CL}_{2}$ y tratado con $\mathrm{H}_{2} \mathrm{SO}_{4} 0,5 \mathrm{~N}$ obteniéndose la fase orgánica $\left(\mathrm{B}_{1}\right)$ y una fase acuosa-ácida la cual es alcalinizada con $\mathrm{NH}_{3}$ al $5 \%$ hasta $\mathrm{pH}$ 9-10, luego es extraída L/L con $\mathrm{CH}_{2} \mathrm{Cl}_{2}$, los alcaloides en forma de base pasan a la fase orgánica. Las soluciones diclorometánicas reunidas son lavadas y secadas. El residuo $\left(\mathrm{B}_{2}\right)$ es considerado el residuo de Alcaloides Totales (AT) cuyo peso es de 13,5 mg.

- Valoración cuantitativa de alcaloides a partir del residuo (B): Protometria. Una alícuota de este residuo $B_{2}$ de $A T$ (6,5 $\mathrm{mg}$ ) fue valorada mediante protometria en medio no acuoso siguiendo el procedimiento de la Farmacopea Europea, antes indicado.

El residuo es disuelto con $20 \mathrm{ml}$ de $\mathrm{AcOH}$, se tituló con una solución de $\mathrm{HClO}_{4}$ 0,01N en $\mathrm{AcOH}$ anhidro en presencia de una gota de violeta de metilo. En la curva de calibrado, $1 \mathrm{ml}$ de solución $0,01 \mathrm{~N}$ de $\mathrm{HClO}_{4}$ indica la presencia de $0,00289 \mathrm{~g}$ de alcaloides. Con la alícuota $\mathrm{B}_{2}$ se utilizaron 2 $\mathrm{ml} \mathrm{de} \mathrm{HClO}_{4}$ que corresponde a un rendimiento de $0,012106 \%$ de AT (Fig. 1 ).

- Alcaloides de la torta de sacha inchi: se pulverizó y humectó $300 \mathrm{~g}$ de torta con $\mathrm{NH}_{3}$ al 5\%, se extrajo por Soxhlet y se trabajó a polaridad creciente obteniéndose $157 \mathrm{~g}$ de residuo seco (ED-T-26). Debido a que esta muestra es grasa, se tomó una alícuota del residuo $(47,9 \mathrm{~g})$ y fue disuelto con $\mathrm{MeOH}+20 \%$ de agua, lavándose con hexano, se obtuvo una Fase Hexánica $(\mathrm{H})$, que luego se extrajo $\mathrm{L} / \mathrm{L}$ con $\mathrm{H}_{2} \mathrm{SO}_{4} 0,5 \mathrm{~N}$, produciendo la Fase Orgánica $\left(\mathrm{H}_{1}\right)$ se alcaliniza con $\mathrm{NH}_{3}$ al 5\% hasta $\mathrm{pH} 9-10$, se extrajo con $\mathrm{CH}_{2} \mathrm{Cl}_{2}$ obteniéndose el residuo $\left(\mathrm{H}_{2}\right)$. La Fase Metanólica-acuosa (M), se alcaliniza, evapora y extrae con $\mathrm{CH}_{2} \mathrm{Cl}_{2}$, se obtiene la Fase Orgánica, que da lugar al residuo $\left(\mathrm{M}_{1}\right)$ de $836 \mathrm{mg}$ y la fase alcalina acuosa $\mathrm{M}_{2}$.

- Detección cualitativa de alcaloides de la torta: 


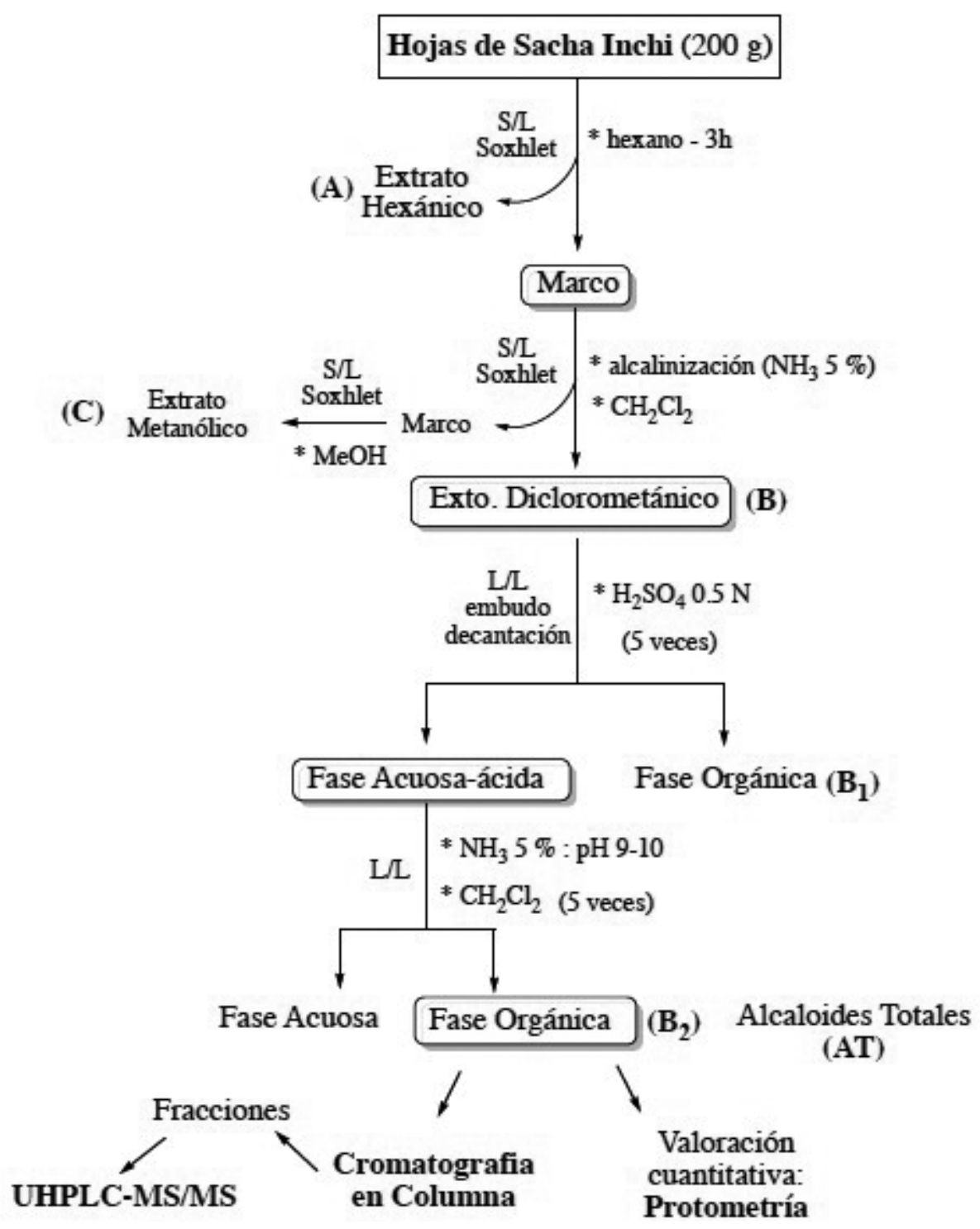

Figura 1. Proceso de extracción de alcaloides en las hojas de sacha inchi Plukenetia volubilis L.

los residuos $\left(\mathrm{H}_{1}\right),\left(\mathrm{H}_{2}\right),\left(\mathrm{M}_{1}\right)$ y $\left(\mathrm{M}_{2}\right)$ y el Marco $(\mathrm{A})$, fueron tratados con sales de metales pesados; el extracto $\left(\mathrm{M}_{1}\right)$ obtenido con $\mathrm{CH}_{2} \mathrm{Cl}_{2}$ fue positivo a la prueba de alcaloides y a Cromatografía en Capa Fina (CCF).

- Valoración cuantitativa de alcaloides a partir del residuo $\left(\mathrm{M}_{1}\right)$ Ponderal: el residuo $\left(\mathrm{M}_{1}\right)$, disuelto en $\mathrm{CH}_{2} \mathrm{Cl}_{2}$, y tratado con $\mathrm{H}_{2} \mathrm{SO}_{4} 0.5 \mathrm{~N}$, produjo la fase $\mathrm{M}_{3}$ orgánica que fue negativa y una fase acuosa-ácida previamente alcalinizada con $\mathrm{NH}_{3}$ al 5\% que fue llevada a pH 9-10 y se extrajo con $\mathrm{CH}_{2} \mathrm{Cl}_{2}$. Las soluciones diclorometánicas produjeron el residuo $\left(\mathrm{M}_{4}\right)$ donde se encuentran los Alcaloides Totales (AT), cuyo pesó es de 18,46 mg (ED-T-26) con un rendimiento de 0,03853\% del extracto orgánico (ED-T-26).

Sin embargo, se debe considerar el rendimiento en peso de $300 \mathrm{~g}$ de Torta $=$ $0,02016 \%$ de AT, y de la semilla de sacha inchi debido a que la torta tuvo que ser desengrasada 


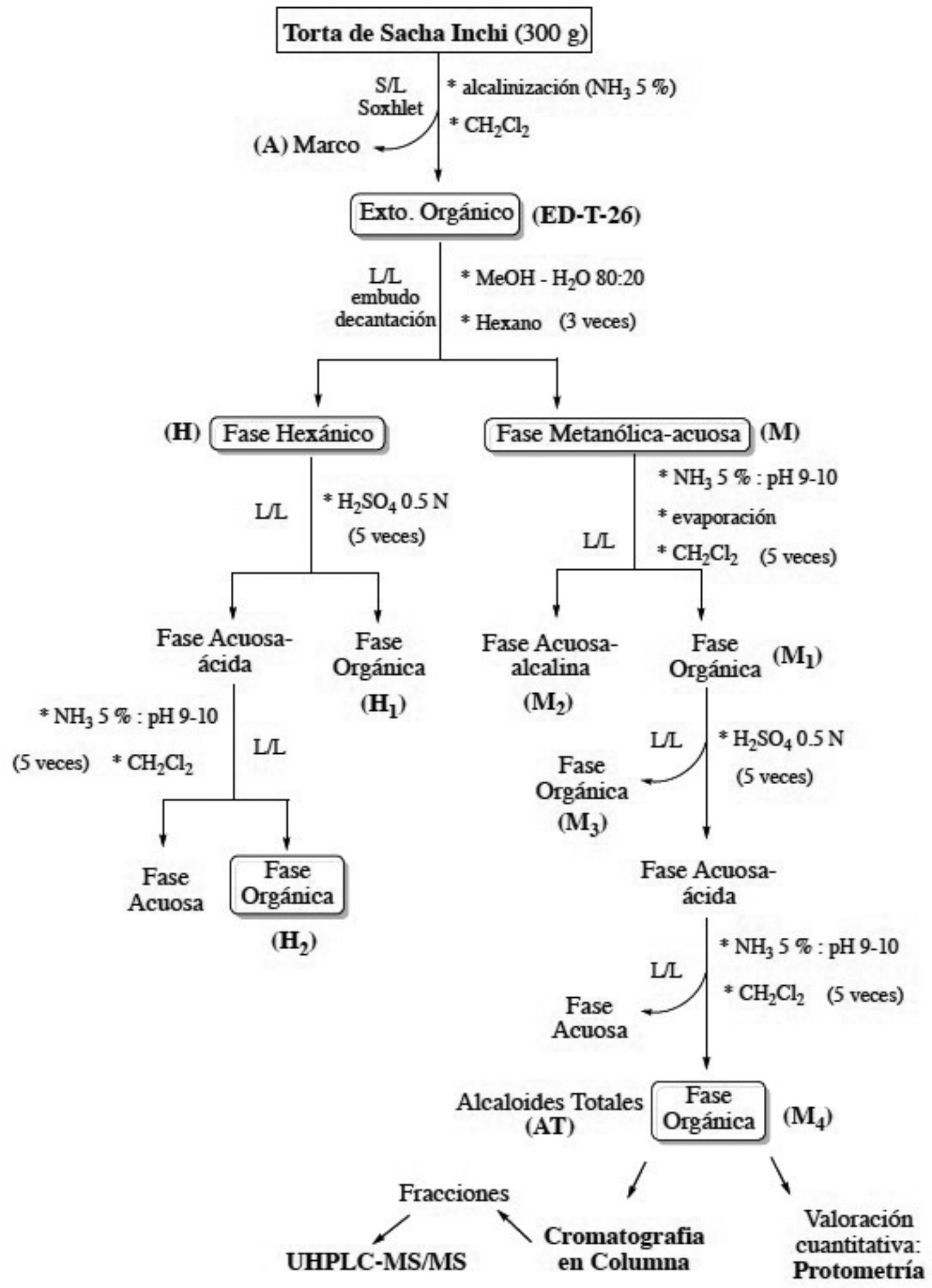

Figura 2. Proceso de extracción de alcaloides en la torta de sacha inchi Plukenetia volubilis L. 
(Torta $+40 \%$ de grasa obtenida directamente por presión) = 0,012101\% de AT.

- Valoración Cuantitativa de Alcaloides, a partir del residuo $\left(\mathrm{M}_{4}\right)$ mediante Protometría: Se evaluó una alícuota del residuo $\left(\mathrm{M}_{4}\right)$ de $\mathrm{AT}, 4,8$ mg, fueron tratados en medio no acuoso de acuerdo con el procedimiento para Alcaloides Tropánicos de Solanaceae en la Farmacopea Europea.

El residuo se disolvió en $20 \mathrm{ml}$ de $\mathrm{AcOH}$ y se tituló con una solución de $\mathrm{HClO}_{4}$ 0,01 N en $\mathrm{AcOH}$ anhidro en presencia de una gota de violeta de metilo. En la curva de calibración, 1 ml de solución de $\mathrm{HClO}_{4}$ 0,01 $\mathrm{N}$ corresponde a la presencia de 0,00289 g de alcaloides. La alícuota de $\left(\mathrm{M}_{4}\right)$ se trató con 0,8 ml de solución de $\mathrm{HClO}_{4} 0,01 \mathrm{~N}$, obteniendo un rendimiento de la torta de 0,009715\% de AT. El rendimiento de semilla de sacha inchi es de: torta $+40 \%$ de grasa previamente separada por presión $=0,005833 \%$ de AT (Fig. 2)

Aislamiento de alcaloides de hojas y torta de sacha inchi: Una alícuota (12 mg) del residuo $\left(B_{2}\right)$ considerado residuo de AT de hojas de sacha inchi. Se purificó mediante cromatografía en columna ultrarrápida en gel de sílice (CC), fue eluido con $\mathrm{CH}_{2} \mathrm{Cl}_{2}-\mathrm{MeOH}$ en proporciones 90:10, 80:20 y 50:50. La Fracción [IV] de este procedimiento de $B_{2}=2,7 \mathrm{mg}$, dio positivo a las reacciones de detección de alcaloides.

Seguidamente se tomó una alícuota (300 mg) del extracto procedente de la torta $\left(\mathrm{M}_{1}\right)$ obtenido con $\mathrm{CH}_{2} \mathrm{Cl}_{2}$, denominado residuo (ED-T-26), fue purificado mediante gel de sílice flash CC, y eluido con Hexano-AcOEt 60: 40 y $\mathrm{CH}_{2} \mathrm{Cl}_{2}-\mathrm{MeOH}$ 90:10. De $\left(\mathrm{M}_{1}\right)$, obteniendo la Fracción [3], 1,5 mg y Fracción [4], 1,3 mg, dieron positivo a las reacciones de detección de alcaloides.

Las muestras obtenidas fueron analizadas: UHPLC-PDA-MS/MS (Shimadzu, Kyoto, Japón) sistema analítico de ultra-alta resolución cromatográfica (UHPLC) con inyector automático
Sil-20ACXR y bomba LC-30AD (Shimadzu), acoplado a un detector de matriz de fotodiodos SPD-M20 (rango 200-800 nm, Shimadzu) y un detector de masas en tándem triple cuadrupolo (MS/MS) LC-8040 (Shimadzu), equipado con una fuente de ionización por electrospray (ESI) en modo de adquisición positivo.

El método UHPLC, fue metanol al 85\% en agua con ácido fórmico al 0,1\% (isocrático), a un flujo de $0,5 \mathrm{~mL} / \mathrm{min}$ y utilizando una columna C18 Kinetex Evo (150 x 4,6 mm x 2,6 $\mu \mathrm{m}$, Phenomenex, California, EE. UU.) con precolumna. El volumen de inyección fue $=0,7 \mu \mathrm{L}$, y en un rango m/z 150500.

La detección de oleamida se realizó en modo ESI positivo, que nos permite identificar los iones de oleamida $\mathrm{m} / \mathrm{z} 282[\mathrm{M}+\mathrm{H}]^{+}$y $304[\mathrm{M}+\mathrm{Na}]^{+}$ $\left(\mathrm{t}_{\mathrm{R}}=4,66 \mathrm{~min}\right)$ (Fig. 3)

Siendo la Oleamida el único compuesto identificado y asimismo es un compuesto que está presente en la industria de aditivos de plástico como agente antideslizante, cabe resaltar que para eliminar la posibilidad de contaminación por la presencia de compuestos relacionados en el plástico se evitó que la muestra se mantenga en contacto con cualquier plástico desde su envio y durante el proceso de aislamiento Garrido-López et al. (2006).

\section{RESULTADOS}

En la fracción [IV] - (B2) de las hojas y la fracción [3] - (M1) de la torta del sacha inchi, mostró un compuesto nitrogenado con un pico molecular $\mathrm{m} / \mathrm{z}=281[\mathrm{M}+]$, que se identifica como 9-octadecenamida (oleamida), cuyo espectro de masas se contrasta con los espectros almacenados en la memoria de la biblioteca espectral.

Paraverificarla estructura química se utilizaron otras técnicas como GC-MS [Espectrómetro de masas Agilent 5977A con analizador cuadrupolar 

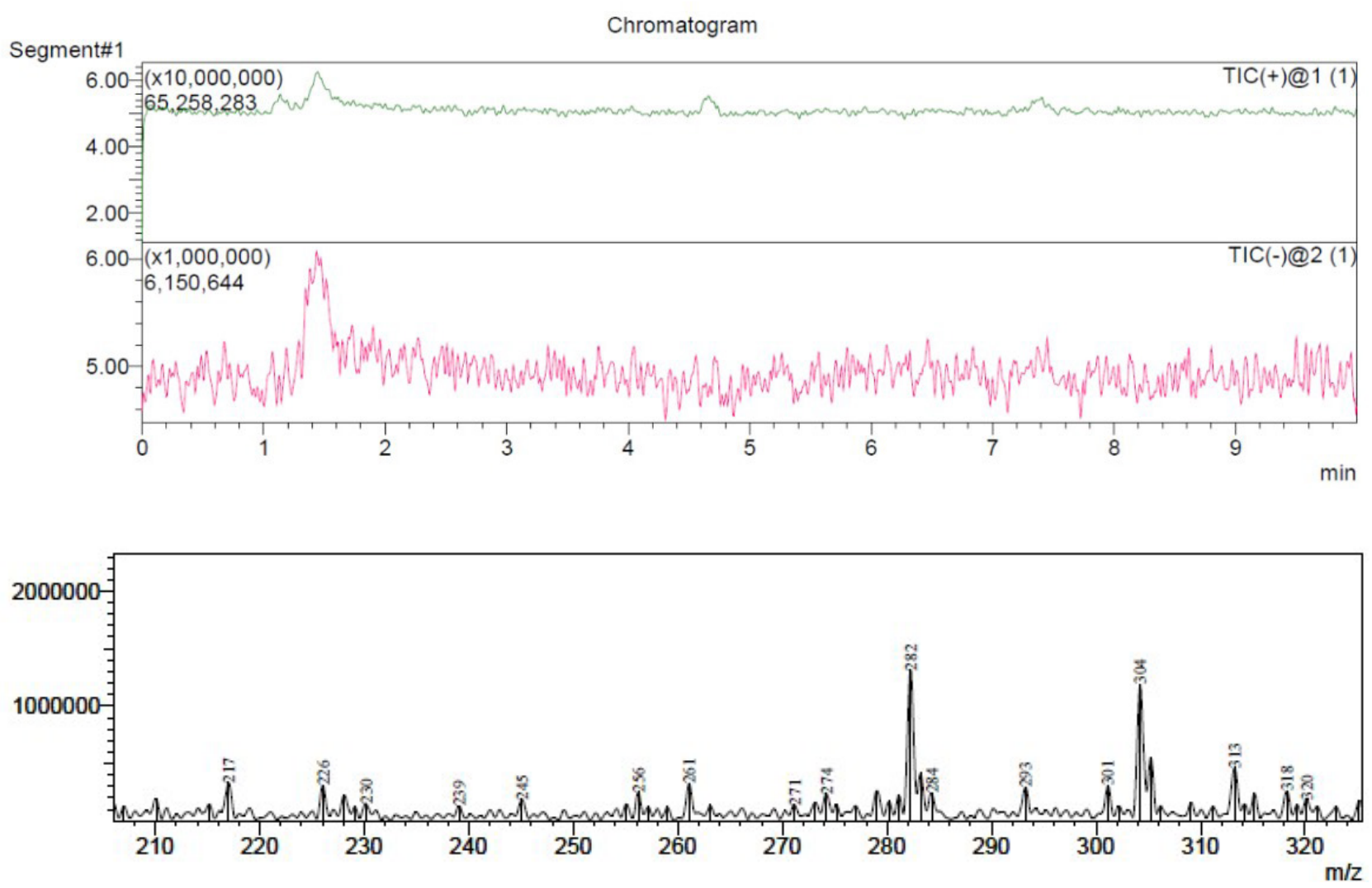

Figura 2. Espectros de masas extraídos de los cromatogramas en modo Scan+ y Scan para Oleamida $\left(t_{R}=4.66\right.$ min ) obtenido del análisis de una muestra mediante UHPLC MS/MS.

de baja resolución, con cromatógrafo de gases (Agilent 7890B)] esta técnica reportó la 9-Octadecenamide, (Z) - C18H35NO, masa 281 a tiempo de retención 24,91 min; ambas técnicas han permitido detectar y cuantificar a la oleamida como componente en las semillas de sacha inchi. El contenido de oleamida presente en hojas es de $0,0015 \%$ y $0,0004 \%$ en semillas.

\section{DISCUSIÓN}

La espectrometría de masas triple cuadrupolo es el método de elección para identificación y cuantificación reproducible de analitos de nivel de trazas en muestras complejas, está en el rango de espectroscópica instrumental microanalítica requiere nanomoles o picomoles del analito para proporcionar información característica de la estructura de una molécula como es el peso y los iones moleculares que establecen el patrón de fragmentación de esta. Asimismo, es una técnica aceptada por las agencias alimentarias entre ellas la European Food Safety Authority (EFSA) para la determinación de alcaloides. Dentro de un residuo de AT encontrarnos metabolitos secundarios con distintos grados de basicidad, entre los que podemos incluir los alcaloides, así como a precursores biogenéticos, tales como aminoácidos o cadenas peptídicas de bajo peso molecular entre otros. Hasta el 2018 los investigadores (Mondragon, 2009; Castillo, 
2011; Castaño et al., 2012) realizaron pruebas cualitativas sobre la presencia de alcaloides en la semilla o la torta de sacha inchi. Srichamnong et al. (2018), llevaron a cabo la cuantificación de alcaloides totales en la especie P. volubilis L. cultivada en Tailandia, sin determinar el tipo de alcaloide presente, sin embargo, los alcaloides de amida y amina no reaccionan con el método que se utilizó para este estudio.

En la presente investigación, durante todo el proceso de búsqueda se realizaron pruebas cualitativas, una vez aislada y purificada la porción alcaloídica, el método ha permitido identificar la cis-9,10-octadecenoamida (oleamida) por fragmentación de masas, esta molécula producida de forma endógena a partir del ácido oleico (el ácido graso omega-9) y que se aisló por primera vez en suero humano en 1989 según Fowler (2004). La oleamida actúa como analgésico en varios modelos de dolor experimental, es capaz de activar receptores $\mathrm{CB}_{1}$, bloquear la comunicación intercelular a través de uniones gap y regular el sueño (Cravatt etal.,1996; Leggett et al., 2004; Bradshaw \& Walker, 2005). Además, Correa \& Yesid (1992) mencionaban que los indígenas consumen nueces tostadas de sacha inchi para recuperar las fuerzas y con el aceite frotan sus cuerpos para curar los dolores musculares, recordemos asimismo el contenido de minerales de estas semillas. Asimismo, se ha encontrado oleamida en hojas de Olea europea "olivo", aunque no en todos los cultivares; es usado en el tratamiento de UV-B2, jugando un papel protector según Días et al. (2018). Por otra parte, Vega-Morales et al. (2019) encontraron Oleamida en Cyperus esculentus L. "tiger nut" o "Chufa", comercializada en Europa como harina, además de ser considerada un superalimento. La oleamida forma parte de la composición de Euphausia superba (krill) cuyo extracto lipídico ha sido aprobado como "novel food" en la Unión Europea. De esta manera emerge como un compuesto prometedor en la industria alimenticia, cosmética y farmacéutica según Benitez et al. (2018), además de su uso como aditivo. Es la primera vez que ha sido encontrada en las semillas y en las hojas de Plukenetia volubilis $\mathrm{L}$.

Adicionalmente, la regulación alimenticia estipula que el solicitante que tenga la intención de acceder al mercado europeo, en este caso las empresas comercializadoras, presenten datos analíticos con métodos validados por la presencia de algunos compuestos no deseables que puedan haber sido reportados en la literatura científica. Matsuura (2015); Dreger et al. (2009) mencionan la presencia de algunos alcaloides regulados tales como: tropánicos y pirrizolidinicos en la familia Euphorbiaceae, por esta razón se llevó a cabo un "screening" en laboratorio analítico acreditado cuyo resultado también fue negativo a la presencia de dichos alcaloides.

\section{CONCLUSIÓN}

El único compuesto encontrado en el residuo de alcaloides totales en hojas y semillas tostadas de sacha inchi fue la cis-9,10-octadecenoamida (Oleamida). El contenido de oleamida presente en hojas es de $0,0015 \%$ y $0,0004 \%$ en semillas, no habiendose encontrado un alcaloide del tipo verdadero en la muestra utilizando técnicas aprobadas en la Unión Europea. Se considera que la semilla tostada de sacha inchi (Plukenetia vulubilis L.) es un alimento tradicional, prometedor e inocuo y puede ser comercializado sin causar daño a la salud. Sin embargo, las hojas pueden ser comercializadas con una mayor investigación. 


\section{AGRADECIMIENTO}

Este proyecto fue realizado gracias al apoyo del Programa SeCompetitivo a través de la Cooperación Suiza - SECO en el Perú y Helvetas Perú.

\section{REFERENCIAS BIBLIOGRÁFICAS}

Antúnez de Mayolo, S. 1981. Amui-o: sumo entre semillas oleo-proteicas. Boletín de Lima, 11: 11-15.

Alcivar, J.; Martinez, M.; Lezcario, P.; Scull, I.; Valverde, A. 2020. Technical note on physical-chemical composition of sacha inchi (Plukenetia volubilis) cake. Cuban Journal of Agricultural Science, 54 (1): 19-23.

Benitez, R.; Coronell, C.; Martin, J. 2018. Chemical characterizaction sacha Inchi (Plukenetia volubilis) seed: oleaginosa promising from the colombian Amazon. International Journal of Current Science Research and Review, 1(1): 1-12,

Bondioli, P.; De la-Bella, L.; Rettke, P. 2006. Alpha linolenic acid rich oils. Composition of Plukenetia volubilis (sacha inchi) oil from Peru. La Rivista Italiana Delle Sostanze Grasse, 83: 120-123.

Brack, A. 1999. Diccionario enciclopédico de plantas útiles del Perú. Centro de Estudios Regionales Andinos Bartolomé de las Casas, Cusco. 400pp.

Bradshaw, H.B.; Walker, J.M. 2005. The expanding field of cannabimimetic and related lipid mediators, British Journal of Pharmacology, 144: 459-465. DOI: https://doi.org/10.1038/ sj.bjp.0706093

Bueno-Borjes, L.; Sartin, M.; Carreño, C.; Vilela, S.; Viegas, P.; Bismara, M. 2018. Sacha inchi seeds from sub-tropical cultivation: effects of roasting on antinutrients, antioxidant capacity and oxidative stability. Journal of Food Science and Technology, 55: 4159-4166. DOI: https:// doi.org/10.1007/s13197-018-3345-1

Cai, Z.Q.; Jiao, D.Y.; Tang, S.X.; Dao, Y.B.; Lei, Y.B.; Cai, C.T. 2012. Leaf photosynthesis, growth, and seed chemicals of sacha Inchi plants cultivated along an altitude gradient. Crop Science, 52 (4): 1859-1867. DOI: https://doi. org/10.2135/cropsci2011.10.0571

Castaño, D.L.; Valencia, M.; Murillo, E.; Mendez, J.J; Eras, J. 2012. Composición de ácidos grasos de sacha inchi (Plukenetia volubilis Linneo) y su relación con la bioactividad del vegetal. Revista Chilena de Nutrición, 39(1): 45-52. DOI: $\quad$ http://dx.doi.org/10.4067/S071775182012000100005

Castillo, E.F.; Castillo, S.F.; Reyes, C.E. 2010. Estudio fitoquímico de Plukenetia volubilis L. y su efecto antioxidante en la lipoperoxidación inducida por $\mathrm{Fe} 3+$ /ascorbato en hígado de Rattus rattus var. albinus. UCV-Scientia, 2(1): 11-21.

Chirinos, R.; Zuloeta, G.; Pedreschi, R.; Mignolet, E.; Larondelle, Y.; Campos, D. 2013. Sacha inchi (Plukenetia volubilis) a seed source of polyunsaturated fatty acids, tocopherols, phy-tosterols, phenolic compounds and antioxidant capacity, Food Chemistry, 141: 1732-1739. DOI: https://doi.org/10.1016/j. foodchem.2013.04.078

Corazón-Guivin, M.; Castro-Ruiz, D.; ChoraMacuyama, W.; Rodriguez, A.; Cachique, D.; Del Castillo, D.; Renno, J.F.; Garcia-Davila, C. 2009. Caracterización genética de accesiones sanmartinenses del Banco Nacional de Germoplasma de Sacha Inchi Plukenetia volubilis L. (E.E. El Porvenir - INIA). Folia Amazónica, 18(1-2): 23 - 31. DOI: https://doi. org/10.24841/fa.v18i1-2.276

Correa, J.; Yesid, H. 1992. Plukenetia volubilis. En: Correa, J.; Yesid, H. (Eds.) Especies vegetales promisorias de los países del Convenio Andrés 
Bello Dioscoreaceae - Euphorbiaceae t. 7. Secretaría del Convenio Andrés Bello, Bogotá. p. 577-596.

Cravatt, B.F.; Lerner, R.A.; Boger, D.L. 1996. Structure determination of an endogenous sleep-inducing lipid, cis-9-octadecenamide (oleamide): a synthetic approach to the chemical analysis of trace quantities of a natural product. Journal of American Chemical Society, 118(3): 580-590. DOI: https://doi. org/10.1021/ja9532345

Días, C.; Pinto, D.C.; Correia, C.; MoutinhoPereira, J.; Oliveira, H.; Freitas, H.; Silva, A.M.; Santos, C. 2018. UV-B radiation modulates physiology and lipophilic metabolite profile in Olea europaea. Journal of Plant Physiology, 222: 39-50. DOI: https://doi.org/10.1016/j. jplph.2018.01.004

Doherty, J.; Sangama, I.; Satalaya, A.; López de Hoyos, G.; Lozano, F. 2007. Diccionario del Quechua de San Martín. Academia Regional de Kechwa de San Martín, Lamas. 78pp

Dostert, N.; Roque, J.; Brokamp, G.; Cano, A.; La Torre, I.; Weigend, M. 2013. Siete especies de plantas vasculares de importancia económica en el Perú: Fichas botánicas. Arnaldoa, 20(2): 359-431.

Dreger, M.; Stanislawska, M.; Krajewska-Patan, A.; Mielcarek, S. 2009. Pyrrolizidine alkaloidschemistry,biosynthesis, pathway, toxicity, safety and perspectives of medicinal usage. Herba Polinca Journal, 55(4): 127-147

EFSA Panel on Contaminants in the Food Chain (CONTAM). 2011. Scientific opinion on Pyrrolizidine alkaloids in food and feed. EFSA Journal, 9(11): 2406. DOI: https://doi. org/10.2903/j.efsa. 2011.2406

EFSA Panel on Contaminants in the Food Chain (CONTAM). 2013. Scientific opinion on Tropane alkaloids in food and feed. EFSA Journal, 11(10): 3386. DOI: https://doi. org/10.2903/j.efsa.2013.3386
Fanali, C.; Dugo, L.; Cacciola, F.; Beccaria, M.; Grasso, S.; Dachà, M.; Dugo, P.; Mondello, L. 2011. Chemical characterization of Sacha Inchi (Plukenetia volubilis L.) oil.Journal Agricultural and Food Chemistry. 59(24): 13043-13049. DOI: https://doi.org/10.1021/jf203184y

Follegatti-Romero, L.A.; Piantino, C.R.; Grimaldi, R.; Cabral, F.A. 2009. Supercritical CO2 extraction of omega-3 rich oil from Sacha inchi (Plukenetia volubilis L.) seeds. Journal of Supercritical Fluids, 49: 323-329.

Fowler, C.J. 2004. Oleamide: a member of endocannabinoid family. British Journal of Pharmacological, 141: 195-196. DOI: https:// doi.org/10.1038/sj.bjp.0705608

Garcilazo de la Vega.1906. Los comentarios reales de los incas (MDCIX). Biblioteca Peruana, Lima. 515pp.

Garrido-López, A.; Esquiu, V.; Tena, M. 2006. Determination of oleamide and erucamide in polyethylene films by pressurized fluid extraction and gas chromatography. Journal of Chromatography A. 1124(1-2): 51-56. DOI: https://doi.org/10.1016/j. chroma.2006.04.086

Gillespie, I. 1993. A sinopsis of neotropical Plukenetia (Euphorbiaceae) including two new species. Systematic Botany, 18(4): 575592. DOI: https://doi.org/10.2307/2419535

GRAS Notice. GRN N ${ }^{\circ}$ 506. 2014. Sacha inchi oil, a vegetable oil derived from Plukenetia volubilis. International Trade Centre. (https:// www.intracen.org/news/GRAS-assessmentdossier-prepared-for-Peruvian-Sacha-Inchi/). Acceso: 19/03/2020

Gutierrez, L.; Rosada, L.; Jiménez,A. 2011.Chemical composition of sacha inchi (Plukenetia volubilis L.) seeds and characteristics of their lipid fraction. Grasas y Aceites, 62: 76-83.

Hamaker, B.; Valles, C.; Gilman, R.; Hardmeier, R.; Clark, D.; Garcia, H.; Gonzales, A.; Kohlstad, I.; Castro, M.; Valdivia, R.; Rodriguez, T.; Lescano, 
M. 1992. Amino acid and fatty acid profiles of the inca peanut (Plukenetia volubilis). Cereal Chemistry, 69(4): 461-463.

Hegnauer, R. 1963. The taxonomic significance of alkaloids. Chemical plant taxonomy. Academic Press, London. 543pp. DOI: https://doi. org/10.1016/B978-0-12-395540-1.50018-X

Leggett, J.; Aspley, S.; Beckett, S.; Dantona, A.; Kendall, D. 2004. Oleamide is a selective endogenous agonist of rat and human CBl cannabinoid receptors, British Journal of Pharmacology, 141(2): 253-262. DOI: https:// doi.org/10.1038/sj.bjp.0705607

Matsuura, H.; Fett-Neto, A. 2015. Plant alkaloids: main features, toxicity, and mechanisms of action. In: Gopalakrishnakone P.; Carlini C.; Ligabue-Braun R. (eds) Plant toxins. Toxinology. Springer, Dordrecht. p. 1-15. DOI: https://doi.org/10.1007/978-94-007-67287_2-1

Mcbride, J. 1951. Flora of Peru: Euphorbiaceae, spurge family Part 3A, no.1. Field Museum of Natural History, Chicago. 288pp.

Mendonça-Franqueiro, E.; Alves-Paiva, R.; Sartim, M.; Callejon, D.; Paiva, H.; Antonucci, G.; Rosa, J.; Cintra, A.; Franco, J.; Arantes, E.; Dias-Baruffi, M.; Sampaio, S. 2011. Isolation, functional, and partial biochemical characterization of galatrox, an acidic lectin from Bothrops atrox snake venom. Acta Biochimica Biophysica Sininica, 43(3):181-92. DOI : https://doi. org/10.1093/abbs/gmr003

Mondragón, G. 2009. Estudio farmacognóstico y bromatológico de los residuos industriales de la extracción del aceite de Plukenetia volubilis $L$. (Sacha inchi). Tesis de pre-grado, Universidad Nacional Mayor de San Marcos, Facultad de Farmacia y Bioquímica, Lima - Peru. 121pp.
Nowak, T.; Haywood, P.; Barondes, S. 1976. Developmentally regulated lectin in embryonic chick muscle and a myogenic cell line. Biochemical Biophys Research Communications, 68(3): 650-657. DOI: https:// doi.org/10.1016/0006-291x(76)91195-5

Ruiz, C.; Diaz, C.; Anaya, J.; Rojas, R. 2013. Análisis proximal, anti-nutrientes, perfil de ácidos grasos y de aminoácidos de semillas y tortas de 2 especies de sacha inchi (Plukenetia volubilis y Plukenetia huayllabambana). Revista de la Sociedad Química del Perú, 79 (1): 29-36.

Sathe, S.; Hamaker, B.; Sze-Tao, K.; Venkatachalam, M. 2002. Isolation, purification, and biochemical characterization of a novel watersoluble protein from inca peanut (Plukenetia volubilis L.). Journal Agricultural Food Chemistry, 50(17): 4906-4908. DOI: https:// doi.org/10.1021/jf020126a

Seigler, D. 1994. Phytochemistry and sistematic of the Euphorbiaceae. Annals Missouri Botanical Garden. 81: 380-401.

Shamsa, F.; Monsef, H.; Ghamooshi, R.; Verdian-riz, M. 2008. Spectrophotometric determination of total alkaloids in some Iranian medicinal plants. Thai Journal of Pharmaceutical Sciences, 32: 17-20.

Srichamnong, W.; Pisamai, T.; Pornsiri, P.; Onanong, N.; Piya T. 2018. Safety assessment of Plukenetia volubilis (inca peanut) seeds, leaves, and their products. Food Science \& Nutrition, 6(4): 962-969. DOI: https://doi. org/10.1002/fsn3.633

Vega-Morales, T.; Mateos-Diaz, C.; Machin, R.; Wiebe, J. 2019. Chemical composition of industrially and laboratory processed Cyperus esculentus rhizomes. Food Chemistry, 297: 124896. DOI: https://doi.org/10.1016/j. foodchem.2019.05.170

\section{Recibido: 6 de mayo de 2021 Aceptado para publicación: 25 de junio de 2021}

